British Journal of Nutrition (2021), 126, 1017-1027

doi: $10.1017 / \mathrm{S} 000711452000495 \mathrm{X}$

(C) The Author(s), 2020. Published by Cambridge University Press on behalf of The Nutrition Society. This is an Open Access article, distributed under the terms of the Creative Commons Attribution licence (http://creativecommons.org/licenses/by/4.0/), which permits unrestricted re-use, distribution, and reproduction in any medium, provided the original work is properly cited.

\title{
Differential associations between a priori diet quality scores and markers of cardiovascular health in women: cross-sectional analyses from TwinsUK
}

\author{
Olatz Mompeo ${ }^{1} \dagger$, Sarah E. Berry ${ }^{2} \dagger$, Tim D. Spector ${ }^{1}$, Cristina Menni ${ }^{1}$, Massimo Mangino ${ }^{1,3 *} \dagger$ and \\ Rachel Gibson ${ }^{2 *}{ }^{2}$ \\ ${ }^{1}$ Department of Twin Research and Genetic Epidemiology, King's College London, London SE1 7EH, UK \\ ${ }^{2}$ Department of Nutritional Sciences, King's College London, London SE1 9NH, UK \\ ${ }^{3}$ NIHR Biomedical Research Centre, Guy's and St Thomas' Foundation Trust, London SE1 9RT, UK \\ (Submitted 11 August 2020 - Final revision received 27 October 2020 - Accepted 2 December 2020 - First published online 10 December 2020 )
}

\section{Abstract}

CVD is the leading cause of death worldwide and, after dementia, is the second biggest cause of death for women. In England, it accounts for one in four of all deaths. Lifestyle modifications represent the primary route both to reduce CVD risk factors and prevent CVD outcomes. Diet constitutes one of the key modifiable risk factors in the aetiology of CVD. We investigated the relationship between nine main dietary indices and a comprehensive range of CVD risk factors in 2590 women from TwinsUK. After adjustment for multiple testing, we found that the Dietary Approaches to Stop Hypertension (DASH) diet was inversely correlated with some of the most common CVD risk factors (BMI, visceral fat (VF), TAG, insulin, homoeostasis model assessment of insulin resistance (HOMA2-IR) and atherosclerotic CVD (ASCVD) risk) with $P_{\mathrm{FDR}}$ ranging from $6.28 \times 10^{-7}$ to $5.63 \times 10^{-4}$. Similar association patterns were detected across most of the dietary indices analysed. In our post hoc investigation, to determine if any specific food groups were driving associations between the DASH score and markers of cardiometabolic risk, we found that increased BMI, VF, HOMA2-IR, ASCVD risk, insulin and TAG levels were directly correlated with red meat consumption $\left(P_{\mathrm{FDR}}\right.$ ranging from $4.65 \times 10^{-9}$ to $\left.7.98 \times 10^{-3}\right)$ and inversely correlated with whole-grain cereal consumption $\left(P_{\mathrm{FDR}}\right.$ ranging from $1.26 \times 10^{-6}$ to $\left.8 \cdot 28 \times 10^{-3}\right)$. Our findings revealed that the DASH diet is associated with a more favourable CVD risk profile, suggesting that this diet may be a candidate dietary pattern to supplement current UK dietary recommendations for CVD prevention.

Key words: Dietary indexes: CVD: Dietary Approaches to Stop Hypertension: Lipid levels

CVD in the UK account for approximately 170000 deaths per year ${ }^{(1)}$. CVD prevalence is higher in males when compared with pre-menopausal women ${ }^{(2)}$. However, it has been observed that in post-menopausal women ( $45-54$ years), CVD prevalence is higher than men $^{(3)}$. Differential patterns have been observed for CVD risk factors between men and women. Evidence suggests that the type 2 diabetic phenotype is associated with a $50 \%$ higher relative risk of CVD amongst women compared with men ${ }^{(4)}$. Prospective cohort studies have suggested that nonfasting blood TAG is a significant CVD risk factor with an approximately 2-fold greater predictive ability in women $v$. men ${ }^{(5,6)}$. A recent review highlighted these sex disparities and recommended the need for a targeted approach in understanding the biological differences in CVD risk in women ${ }^{(7)}$.
Modifying exposure to lifestyle factors reduces CVD risk factors and represents the primary route to prevent CVD outcomes. Diet is a key modifiable lifestyle factor in the aetiology of $\mathrm{CVD}^{(8)}$. Traditionally, research in this area has focused on individual macronutrients, such as fats and carbohydrates. However, we consume a mixture of foods rather than single nutrients. In recent years, nutritional science has shifted from reductionist approach of single nutrients towards investigation of combinations of foods and nutrients ${ }^{(9)}$. The combinations of foods consumed may, in part, be responsible for health effects through nutrient-nutrient interactions.

Combinations of foods defined a priori (referred as dietary 'patterns') are increasingly reported in nutritional epidemiological literature in relation to CVD outcomes ${ }^{(10)}$. Dietary patterns

Abbreviations: A-Med, Amended Mediterranean Score; ASCVD, atherosclerotic CVD; DASH, Dietary Approaches to Stop Hypertension; DRV, Dietary Reference Value; FDR, false discovery rate; HDI, Healthy Diet Indicator; HEI, Healthy Eating Index 2010; HOMA2-IR, homeostasis model assessment of insulin resistance; IMD, index of multiple deprivation; O-Med, Original Mediterranean Score; VF, visceral fat.

*Corresponding authors: Rachel Gibson, email rachel.gibson@kcl.ac.uk; Massimo Mangino, email massimo.mangino@kcl.ac.uk

$\uparrow$ These authors contributed equally to this work. 
such as the Mediterranean and the Dietary Approaches to Stop Hypertension (DASH) diet have been adopted into public health recommendations in the USA ${ }^{(11)}$. It has been suggested that basing nutrition guidelines around dietary patterns may be more readily translatable by the public ${ }^{(12)}$. To date, UK public health dietary guidelines do not include any dietary pattern recommendations.

Although improved eating patterns may have important effects on reducing population-wide markers of CVD risk, the evidence supporting the population-wide efficacy of applying dietary pattern guidelines is limited. A recent review found thirty-one different scores applied in nutritional epidemiological studies testing associations between diet patterns and CVD-related outcomes ${ }^{(10)}$. From the nine most commonly applied scores, four had been applied in UK population groups and most studies testing associations of one or two scores against a limited range of CVD health outcomes ${ }^{(13)}$. Simultaneously testing a set of dietary patterns against a standard set of CVD risk markers will inform candidate dietary patterns to be taken forward for testing in clinical trials.

The main aim of this study is to investigate the relationship between nine main dietary indices ${ }^{(10)}$ and a comprehensive range of CVD risk factors including: estimated atherosclerotic CVD (ASCVD) risk, anthropometric (BMI and visceral fat (VF)), lipid profile (TAG, LDL, HDL, total cholesterol), vascular function (pulse wave velocity, blood pressure) and glucose metabolism (insulin, glucose and homoeostasis model assessment of insulin resistance (HOMA2-IR)). From our primary analyses, we identified the most favourable dietary pattern to take forward for post hoc analyses to investigate dose-response associations and to investigate if any observed associations between dietary patterns and health outcomes are independent of any individual food group included within the dietary pattern.

\section{Methods}

\section{Study population}

All individuals included in this study are part of the UK Adult Twin Register (TwinsUK). TwinsUK is a large cohort of twins historically developed to study the heritability and genetics of diseases with a higher prevalence among women ${ }^{(14)}$. In this study, we included a total of 2590 female individuals, with an average age of 58.1 (SD 10.2) years. All participants had complete food frequency data and at least one cardiometabolic measurement of interest collected between 2004 and 2010. Although dietary patterns, in particular in woman, are mostly consistent from childhood to adulthood ${ }^{(15)}$, clinical and biochemical measures included in the analysis were selected to be on average within 2.65 (SD 1.52) years from the diet assessment. A detailed flow chart is described in online Supplementary Fig. S1. TwinsUK is approved by NRES Committee London-Westminster (REC ref: EC04/015), and all participants provided informed written consent.

\section{Dietary assessment and dietary score generation}

All participants completed a 131-item FFQ that has been previously validated against pre-nutrient biomarkers in the European
Prospective Investigation into Diet and Cancer Norfolk $^{(16,17)}$. The questionnaire captures average intakes in the past year. Nutrient and energy intakes were determined by FETA (Food Frequency Questionnaire European Prospective Investigation into Cancer and Nutrition Tool for Analysis) software ${ }^{(18)}$. Subjects were excluded from the analysis if more than ten food items were left unanswered in their FFQ or if the total energy intake was more than two SD outside the estimated $\mathrm{BMR}^{(19)}$ (determined by the Harris-Benedict equation ${ }^{(20)}$ ).

The full list of FFQ items included to define food group categories for dietary score calculation is reported in online Supplementary Table S1.

The selection of diet indices included in this study is based on Aljuraiban et $a l^{(10)}$ (Table 1 and online Supplementary Tables S2-S10) and reflects the nine most frequently reported diet indices reported against CVD risk. We selected nine dietary indices: Alternative Healthy Eating Index (HEI) ${ }^{(21)}$, Amended Mediterranean Score (A-Med) ${ }^{(22)}$, Dietary Approaches to Stop Hypertension (DASH) ${ }^{(23)}$, Diet Quality Index International ${ }^{(24)}$, Dietary Reference Values (DRV) ${ }^{(25)}$, Healthy Diet Indicator $(\mathrm{HDI})^{(26)}$, Healthy Eating Index 2010 (HEI $^{(27)}$, Nordic Diet Score ${ }^{(28)}$ and the Original Mediterranean Score (O-Med) ${ }^{(29)}$. All indices were calculated in $\mathrm{R}$ (version 3.6.2) ${ }^{(30)}$.

\section{Measures of cardiovascular health}

For this study we analysed: BMI, VF (g), systolic and diastolic blood pressure $(\mathrm{mmHg})$, pulse wave velocity $(\mathrm{m} / \mathrm{s})$, fasting lipid measures (mmol/l) including HDL, LDL, total cholesterol and TAG, fasting plasma insulin (pmol/l) and glucose (mmol/l), insulin resistance (HOMA2-IR) and ASCVD risk score. A detailed description of every clinical and biochemical measure utilised in this study has been previously reported in detail( ${ }^{(31-33)}$. In brief, BMI was determined from weight and height measurements (weight $(\mathrm{kg}) /$ height $\left(\mathrm{m}^{2}\right)$ ). VF was measured via dual-energy X-ray absorptiometry scans (Hologic QDR; Hologic, Inc.) ${ }^{(33)}$. Peripheral systolic and diastolic BP were measured by a trained nurse who used an automated cuff sphygmomanometer (OMRON HEM713C) with the participant in the seated position for at least $3 \mathrm{~min}$ before taking three measurements. Hypertension status was defined as systolic blood pressure $\geq 140 \mathrm{mmHg}$ and/or diastolic blood pressure $\geq 90 \mathrm{mmHg}$ and/ or treatment with antihypertensive medication. For participants taking antihypertensive drugs, we added $15 \mathrm{mmHg}$ to systolic and $10 \mathrm{mmHg}$ to diastolic blood pressure to correct for the effect of the treatment ${ }^{(34)}$. Arterial stiffness pulse wave velocity was calculated from sequential recordings of electrocardiogramreferenced carotid and femoral pressure waveforms ${ }^{(31)}$. All biochemical assessment (lipid profiles, insulin and glucose levels) levels were measured after a 10-h overnight fasting period. HOMA2-IR was calculated by the HOMA2 online application (https://www.dtu.ox.ac.uk/homacalculator/). Fasting insulin (pmol/l) and glucose (mmol/l) were measured using an enzymatic assays previously described ${ }^{(33,35)}$. Individuals on lipidor blood glucose-lowering medications were excluded from the study. The individual CVD risk was estimated using the 10 -year ASCVD risk score ${ }^{(36)}$. The ASCVD risk score is an algorithm used to estimate the 10-year cardiovascular risk of an 
NS British Journal of Nutrition

Table 1. Summary of the food groups/dietary groups of the dietary indices included in the study

\begin{tabular}{|c|c|c|c|c|c|c|c|c|c|}
\hline \multirow{2}{*}{$\begin{array}{l}\text { Food groups } \\
\text { Dietary groups }\end{array}$} & \multicolumn{9}{|c|}{ Dietary indices } \\
\hline & O-Med & A-Med & A-HEI & $\mathrm{HDI}$ & DASH & DQI-I & NDS & HEI & DRV \\
\hline Vegetables & $\approx$ & $\approx$ & 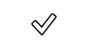 & 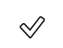 & $\approx$ & $\approx$ & $\approx$ & $\approx$ & $\approx$ \\
\hline Starchy vegetables & $\sim$ & & 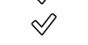 & & & $\sim$ & $\approx$ & & \\
\hline Fruit juice & & $\approx$ & $\approx$ & & 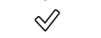 & & $\approx$ & 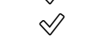 & $\mathscr{q}$ \\
\hline Refined grains & 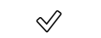 & & & & & 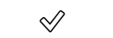 & & 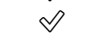 & \\
\hline Whole grains & $\approx$ & 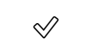 & 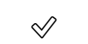 & & 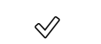 & $\approx$ & $\approx$ & $\approx$ & \\
\hline Legumes & $\approx$ & 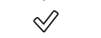 & $\leadsto$ & & 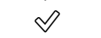 & $\approx$ & & 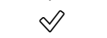 & \\
\hline Nuts & 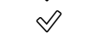 & 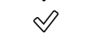 & 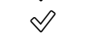 & & $\approx$ & & & 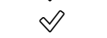 & \\
\hline Tofu & & & $\approx$ & & $\leadsto$ & & & $\mathscr{q}$ & \\
\hline Seeds & & & & & & & & 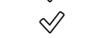 & \\
\hline Seafood & $\approx$ & 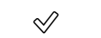 & & & & 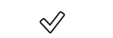 & & 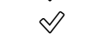 & $\approx$ \\
\hline Poultry & 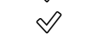 & & & & & 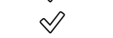 & & 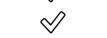 & \\
\hline Red meat & 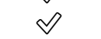 & 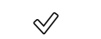 & 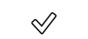 & & 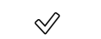 & 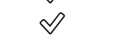 & & 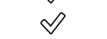 & \\
\hline Processed meat & 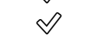 & 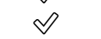 & $\approx$ & & $\approx$ & 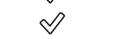 & & 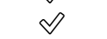 & \\
\hline Eggs & & & & & & 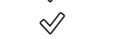 & & 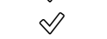 & \\
\hline Butter & $\approx$ & & & & & & $\approx$ & & \\
\hline Dairy products & 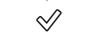 & & & & & 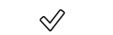 & 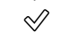 & 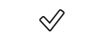 & \\
\hline Low-fat dairy products & 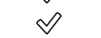 & & & & 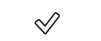 & $\approx$ & $\leadsto$ & $\approx$ & \\
\hline Cheese & $\approx$ & & & & & 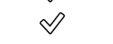 & 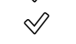 & $\sim$ & \\
\hline Sugar-sweetened beverages & & & 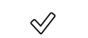 & & & & & & \\
\hline Nutrients & & & & & & & & & \\
\hline Carbohydrates & & & & & & 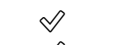 & & & $\approx$ \\
\hline Fibre & & & & $\approx$ & & 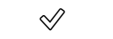 & & & $\approx$ \\
\hline Sugars & & & & $\mathscr{q}$ & & & & & $\mathscr{q}$ \\
\hline Protein & & & & 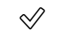 & & $\approx$ & & & \\
\hline Total fat & & & & & & $\mathscr{q}$ & & & 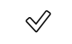 \\
\hline Monounsaturated & $\approx$ & 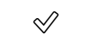 & & & & $\approx$ & & $\approx$ & \\
\hline Polyunsaturated & & & $\approx$ & 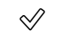 & & 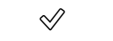 & & 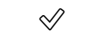 & \\
\hline$n-3$ & & & 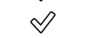 & & & & & & \\
\hline Saturated & $\approx$ & 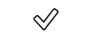 & & 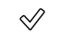 & & 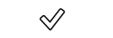 & & 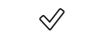 & $\approx$ \\
\hline Trans-fats & & & 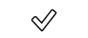 & & & & & & \\
\hline Cholesterol & & & & 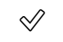 & & $\approx$ & & & \\
\hline $\mathrm{Na}$ & & & $₫$ & & & $\approx$ & & $\approx$ & 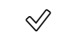 \\
\hline $\mathrm{Fe}$ & & & & & & 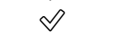 & & & \\
\hline $\mathrm{Ca}$ & & & & & & $\approx$ & & & \\
\hline Vitamin C & & & & & & $\approx$ & & & \\
\hline Alcohol & $\approx$ & 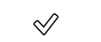 & 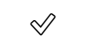 & & & & & & \\
\hline
\end{tabular}

O-Med, Original Mediterranean Score; A-Med, Amended Mediterranean Score; A-HEI, Alternative Healthy Eating Index; DASH, Dietary Approaches to Stop Hypertension; DQI-I, Diet Quality Index International; DRV, Dietary Reference Values; HDI, Healthy Diet Indicator; HEI, Healthy Eating Index 2010; NDS, Nordic Diet Score. 
individual using the individual's sex, ethnicity, age, smoking status, cholesterol levels, blood pressure and diabetes mellitus status. The ASCVD risk score was calculated using the online ASCVD risk estimator (http://tools.acc.org/ASCVD-RiskEstimator-Plus/) $)^{(32)}$.

\section{Assessment of covariates}

All analyses were adjusted for five major confounders: Age, smoking status, physical activity, menopause status and the index of multiple deprivation (IMD). Furthermore, alcohol and energy intakes were included in the analysis models as appropriate. Both alcohol and energy intakes were estimated from the FFQ (mean $\mathrm{g} / \mathrm{d}$ and $\mathrm{kJ}$ for alcohol and energy, respectively $)^{(14)}$. Age, physical activity smoking and menopause status were recorded as part of the self-report lifestyle questionnaire ${ }^{(14)}$. The IMD is a composite decile score measuring the area-level deprivation (lower deciles represent the most deprived areas). IMD was generated combining the data repositories of the four UK's administrative countries: England (IMD version 2015), Scotland (IMD version 2016), Wales (IMD version 2014) and Northern Ireland (IMD version 2017). The IMD includes seven different domains representing income, employment, education, skills and training, health deprivation and disability, crime, barriers to housing and services and living environment deprivation. The IMD was based on the postcode reported by the participant at the time of sample collection ${ }^{(14)}$.

\section{Statistics}

Data distribution for both dietary indices and CVD risk factors was graphically explored in order to assess normality. Since both diet indices and cardiovascular risk factors are reported in different units, in order to compare the results, they were both normalised using a rank-based inverse normal transformation approach $^{(37)}$. All association tests included in this study were performed using linear mixed effects models in $\mathrm{R}$ version 3.6.2 ('Imer' function as part of 'Ime4' package) (38).

We performed a full linear mixed model where standardised CVD risk factors were the response variables and the standardised dietary indices were fixed effects predictors. We included age, smoking, physical activity, IMD, menopause, energy intake (fixed effect) as main covariates. The majority of the data set (94.7\%) was composed by related individuals (both co-twin from each family). Therefore, 'family unit' was also included as covariate (random effect) in the models to account for the twin structure and relatedness. The fit of the full linear regression model was compared with the fit of a null model (which excluded the CVD risk factors as a predictor variable) using the ANOVA function returning the overall $P$ value representing the significance of the difference between the nested models.

Alcohol consumption was included as covariate (fixed effect) in the analyses for those dietary indices that do not take alcohol into account (DASH, Nordic, HDI and DRV) following the recommended methodology (online Supplementary Tables S2-S10) ${ }^{(21-29,39)}$

From these analyses, we identified DASH as the most favourable dietary pattern to take forward for post hoc analyses to test dose response and independence of association against score components.

Similarly, both the quintile analysis and the nutrient analysis were performed using a linear mixed model including CVD risk factors as response variables and DASH quintiles (categorical) and DASH food group or nutrient $(\mathrm{g} / \mathrm{d}$ ) as fixed effect predictors. Age, smoking, physical activity, IMD, menopause, energy intake, alcohol consumption (fixed effect) and family structure (random effects) were added as covariates. In the quintile analysis, the 5 th quintile (representing the 'healthiest' category) was set as baseline.

To test if observed associations between DASH were independent of individual score components, we repeated the analyses with a modified DASH score ${ }^{(40)}$. The food group or nutrient analysis was adjusted for a modified diet score that did not include the respective component along with the included covariates (age, smoking status, physical activity, IMD, energy intake, alcohol consumption and family structure $)^{(41)}$.

To correct for multiple testing, $P$ values were adjusted using the Benjamini and Hochberg method ${ }^{(42)}$. Association tests with $P_{\mathrm{FDR}}<0.01$ were considered statistically significant, while $P_{\mathrm{FDR}}<0.05$ and $\geq 0.01$ were considered 'suggestive'.

\section{Results}

Here, we included 2590 female individuals from TwinsUK. The full descriptive characteristics of the study population are presented in Table 2 . The participants had an average age of 58.1 (sD 10.2) years with $55.7 \%$ of the subjects classified as overweight/obese (BMI $>25 \mathrm{~kg} / \mathrm{m}^{2}$ ). The majority of the participants were post-menopausal (85\%), non-smokers (57\%), not engaged in high physical activity (89\%) and had a good socio-economic

status ( $66 \%$ of the participant at the time of sample collection lived in an area with IMD $\geq 7$ ). Measurement of agreement (Pearson's correlation) between the scores is reported in online Supplementary Fig. S2. Correlation coefficients ranged from -0.09 to 0.85 . We observed the strongest correlations between O-Med and A-Med ( $r$ 0.85), whereas weakest correlation was between HEI and DRV $(r-0 \cdot 09)$.

\section{Dietary scores and CVD measures}

All association results between the dietary indices and CVD risk are presented in Fig. 1 and online Supplementary Table S11.

After adjusting for multiple testing using Benjamini and Hochberg false discovery rate (FDR), we found that the DASH score was correlated with lower BMI, as well as lower VF, TAG, insulin, HOMA2-IR values and decreased ASCVD risk with effects ranging from $\beta=-0.04$ (95\% CI 0.95, 0.98) to $-0 \cdot 12$ ( $95 \%$ CI $0.85,0.92)$ for ASCVD risk and insulin/HOMA2-IR, respectively (Fig. 1 and online Supplementary Table S11). A similar pattern of association was detected across all dietary indices as shown in Fig. 1 and online Supplementary Table S11.

We also observed a positive correlation between HDL levels, DASH, A-Med, DQI-I and O-Med with $\beta$ ranging from 0.08 (95\% CI 1.04, 1·12) for DASH, DQI-I and O-Med to 0.11 (95\% CI $1 \cdot 07$, 1.16) for A-Med (Fig. 1 and online Supplementary Table S11). 
Table 2. Demographic, CVD risk factors and dietary indices characteristics of the participants $(n 2590)$

(Mean values and standard deviations; numbers and percentages)

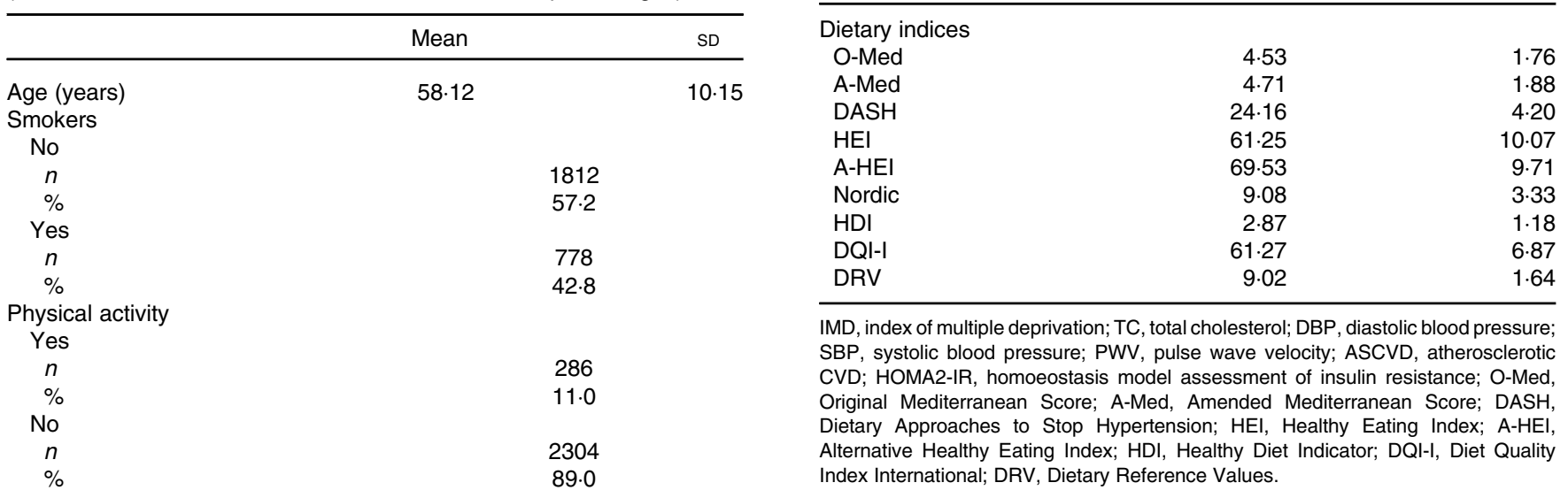

Finally, no association (either statistically significant or suggestive) was observed between DRV and CVD risk factors and (Fig. 1 and online Supplementary Table S11).

We then focused our attention on DASH to investigate if the observed associations with BMI, VF, TAG, HDL, insulin, HOMA2-IR and ASCVD risk were driven by one specific: (a) set of individuals (quintile analysis) and (b) dietary component.

After dividing DASH in quintiles, the analysis showed that the subjects with the less healthy diet style (1st and 2nd quintiles) are driving the association between DASH and BMI, VF, HDL, TAG and ASCVD risk score (Fig. 2 and online Supplementary Table S12). However, HOMA2-IR and insulin levels were consistently different across all quintiles (1st to 4th) when compared with the reference (5th quintile, representing the healthiest individuals) (Fig. 2 and online Supplementary Table S12). Therefore, the associations between DASH and HOMA2-IR and insulin levels were not determined by any specific class of individual.

Two nutrients (red/processed meat and whole-grain cereal) were mostly responsible for the association observed between DASH and CVD risk factors. Our results show that increased red meat consumption was associated with increased BMI $\left(P_{\mathrm{FDR}}=6.03 \times 10^{-6}\right)$, VF $\left(P_{\mathrm{FDR}}=4.65 \times 10^{-9}\right)$, HOMA2-IR $\left(P_{\mathrm{FDR}}=3.69 \times 10^{-3}\right)$, ASCVD risk $\left(P_{\mathrm{FDR}}=4.76 \times 10^{-4}\right)$, insulin $\left(P_{\mathrm{FDR}}=7.98 \times 10^{-3}\right)$ and TAG $\left(P_{\mathrm{FDR}}=3.39 \times 10^{-4}\right)$ levels. Interestingly, there is a suggestive evidence $\left(0.05<P_{\mathrm{FDR}}\right.$ $\leq 0 \cdot 01)$ that increased read meat consumption is associated with lower HDL levels $\left(P_{\mathrm{FDR}}=2.77 \times 10^{-2}\right.$ ) (Fig. 3 and online Supplementary Table S13).

Our results also highlighted that an increased consumption of whole-grain cereal is associated with lower BMI (adj- $P$ value $\left.=8.28 \times 10^{-3}\right), \quad$ ASCVD risk $\quad\left(P_{\mathrm{FDR}}=8.72 \times 10^{-4}\right), \quad \mathrm{VF}$ $\left(P_{\mathrm{FDR}}=1.26 \times 10^{-6}\right)$, HOMA2-IR $\left(P_{\mathrm{FDR}}=3.35 \times 10^{-3}\right)$, insulin $\left(P_{\mathrm{FDR}}=2.30 \times 10^{-3}\right)$ and TAG's levels $\left(P_{\mathrm{FDR}}=8.72 \times 10^{-4}\right)$. Our results also present a suggestive association between increased HDL levels and whole-grain cereal intake $\left(P_{\mathrm{FDR}}=1.75 \times 10^{-2}\right)$ (Fig. 3 and online Supplementary Table S13).

Finally, we also observed a significant reduction of $\mathrm{VF}$ $\left(P_{\mathrm{FDR}}=3.96 \times 10^{-3}\right)$ and TAG levels $\left(P_{\mathrm{FDR}}=5.25 \times 10^{-3}\right)$ associated with increased fruit intake (Fig. 3 and online Supplementary Table S13). 

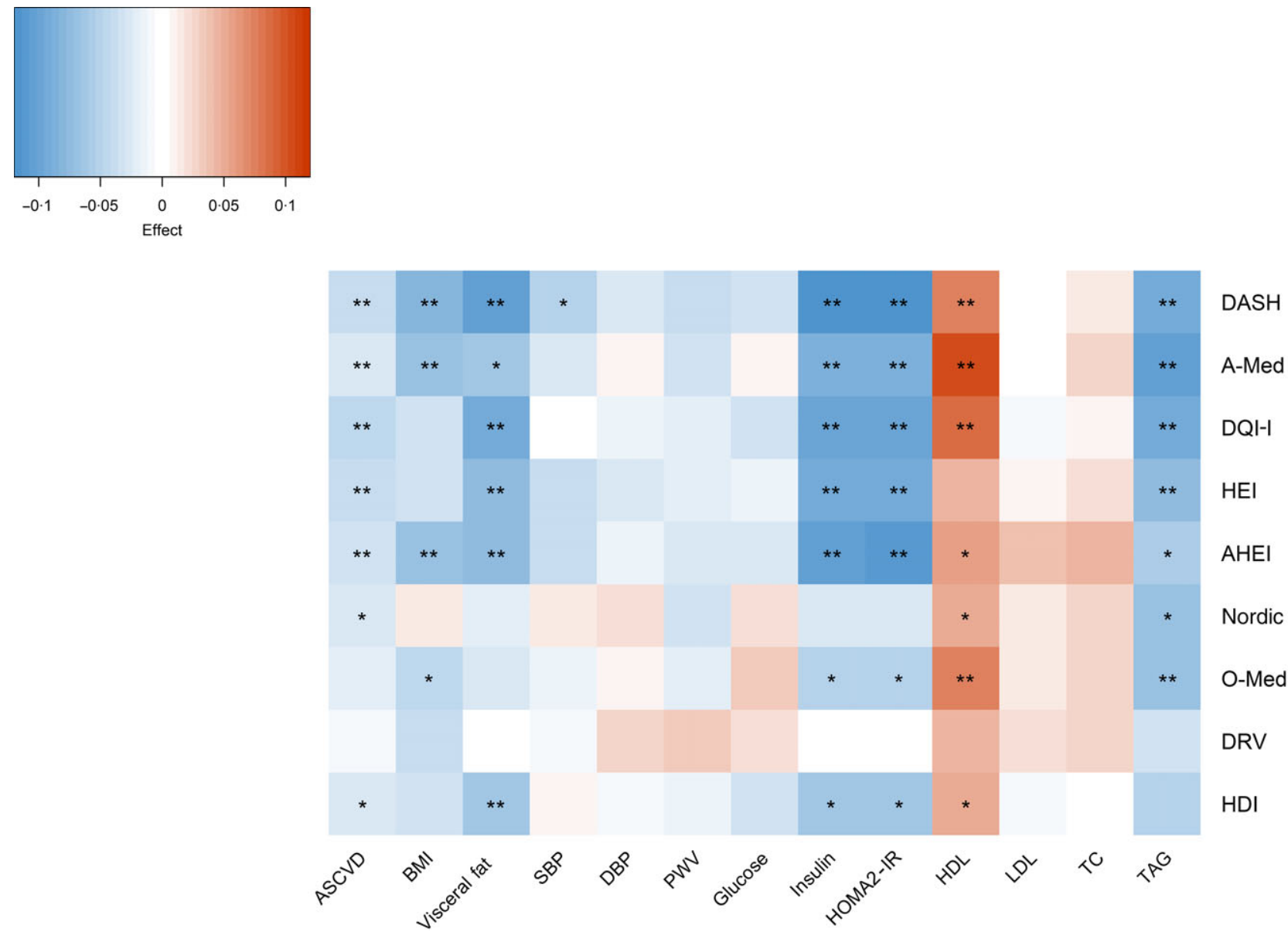

Fig. 1. Heatmap representing the results of the association test between nine main dietary indices and thirteen CVD risk factors. The colour scale illustrates the effect $(\beta)$ of each dietary index on the relative CVD risk factor. Red and blue indicate positive and negative effects, respectively. Colour intensity represents the degree of positive/ negative effect. The asterisks indicate suggestive ( $\left.{ }^{*} P_{\mathrm{FDR}}<0.05\right)$ and significant ( $\left.{ }^{* *} P_{\mathrm{FDR}}<0.01\right)$ Benjamini and Hochberg-adjusted $P$ values. To compare the effect of the indices across the CVD risk factors, both indices and risk factors were standardised ( $z$-score) before the analysis. Age, smoking, physical activity, index of multiple deprivation, menopause, energy intake and family relatedness were included as covariates in all the analyses. DASH, Dietary Approaches to Stop Hypertension; A-Med, Amended Mediterranean Score; DQI-I, Diet Quality Index International; HEI, Healthy Eating Index; A-HEI, Alternative Healthy Eating Index; O-Med, Original Mediterranean Score; DRV, Dietary Reference Values; HDI, Healthy Diet Indicator; ASCVD, atherosclerotic CVD; SBP, systolic blood pressure; DBP, diastolic blood pressure; PWV, pulse wave velocity; HOMA2-IR, homoeostasis model assessment of insulin resistance; TC, total cholesterol.

\section{Discussion}

To our knowledge, this is the first study to investigate the associations between the main a priori dietary indices with multiple objective markers of cardiovascular health in a UK female population sample. Of the nine scores we tested, seven demonstrated benefit against markers of glucose metabolism (insulin and HOMA2-IR) and TAG. These CVD risk markers are considered particularly important to the development of CVD in women ${ }^{(43-45)}$. Therefore, our findings suggest the potential application of a variety of dietary patterns to modify this female-specific risk phenotype. DASH, in particular, showed the greatest number of beneficial associations with CVD risk factors, including measures of adiposity, independent of age, smoking status, daily energy intake and alcohol consumption.

The differential associations we have observed highlight how the components of the various dietary scores - nutrients $v$. foods, and types of foods can influence associations with CVD risk. The majority of the scores we tested (DASH, HEI, Alternative
HEI, DQI-I and A-Med) were developed using American dietary guidelines and/or derived from American population samples ${ }^{(10)}$. As reported in the Dietary Patterns Methods Project, we found moderate to strong correlations between DASH, Alternative HEI, HEI and A-Med ${ }^{(46)}$. The Dietary Patterns Methods Project observed associations between higher DASH, Alternative HEI and A-Med scores with reduced CVD mortality in female only cohorts ${ }^{(46)}$. These findings may represent the common elements of all the scores - whole-grain, fruit and vegetable intake being established as protective against $\mathrm{CVD}^{(47,48)}$. Through testing the diet scores against a range of established CVD risk marker, we observed DASH to have significant benefit against the most markers. These findings are consistent with previous research showing health benefits of following the DASH diet on insulin ${ }^{(49)}$, TAG $^{(50)}$ visceral obesity ${ }^{(51)}$, HOMA2-IR ${ }^{(52)}$ and ASCVD ${ }^{(53)}$. The DASH diet is characterised by high consumption of vegetables, fruit, low-fat dairy products, whole grains, legumes, nuts, and low consumption of red and 
(a)

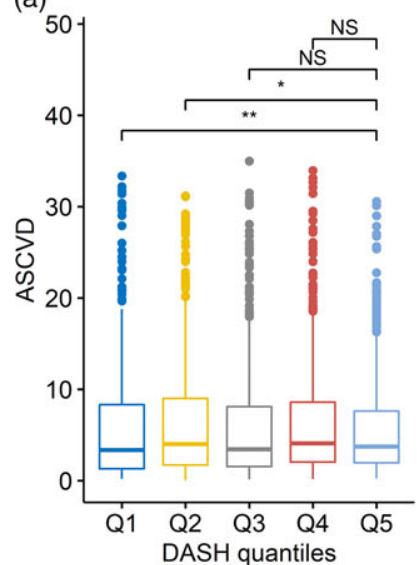

(e) (b)

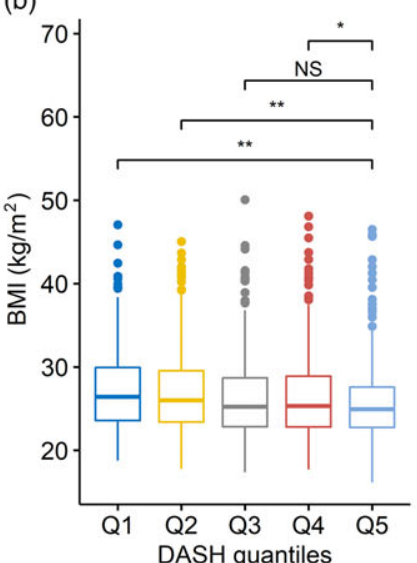

(f) (c)

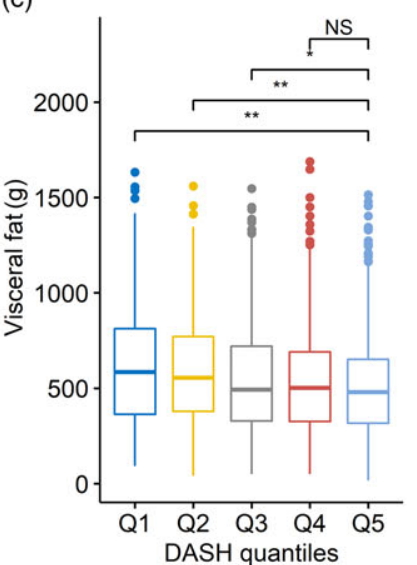

(d)

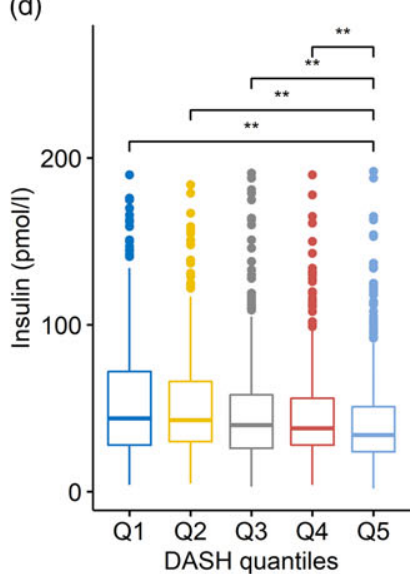

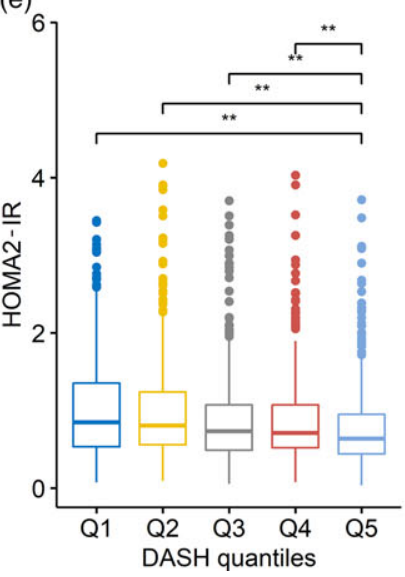

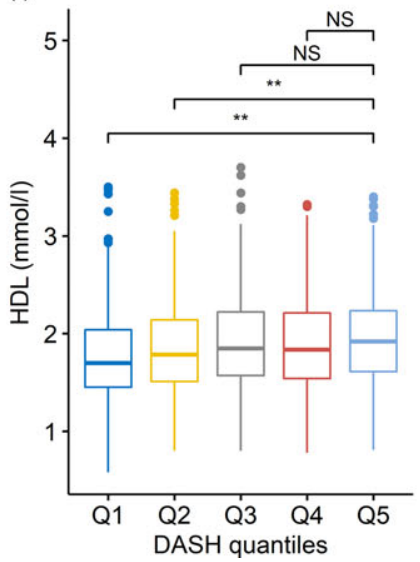

(g)

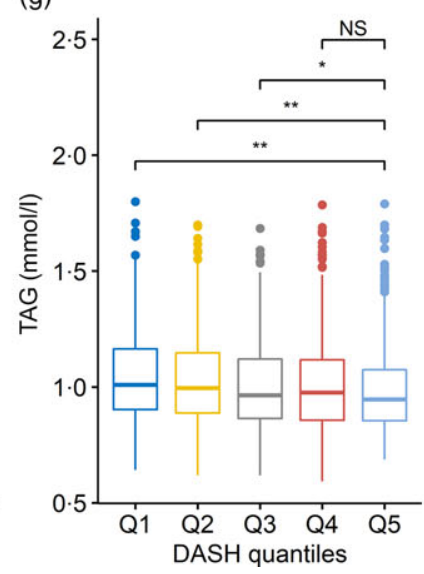

Fig. 2. Each box plot represents the distribution of values for the CVD risk factors across the Dietary Approaches to Stop Hypertension (DASH) quintiles. Boxes represent the median, 25th and 75th percentiles of the distribution of CVD risk factors; whiskers represent the 5th and 95th percentiles of the distribution of CVD risk factors. Linear mixed model including family structure has been used to test a difference of distribution across DASH quintiles. For this analysis, the 5th DASH quintile (representing the healthiest individuals; $n 417$ ) was used as baseline and compared with Q1 ( $n 691)$, Q2 ( $n$ 444), Q3 ( $n$ 469) and Q4 ( $n 569$ ). Suggestive and significant Benjamini and Hochberg-adjusted $P$ values are marked with asterisks $\left({ }^{*} P_{\mathrm{FDR}}<0.05\right.$ and $\left.{ }^{* *} P_{\mathrm{FDR}}<0.01\right)$. NS indicates non-significant differences.

processed meat, $\mathrm{Na}$, and sugar-sweetened beverages. In the present study, DASH was the only score that showed potential beneficial association with blood pressure, a finding in agreement with the DASH feeding trial ${ }^{(41)}$. Potential benefits of the diet have been hypothesised to be due to the diets nutrient profile with high levels of fibre ${ }^{(54)}, \mathrm{Ca}^{(55)}, \mathrm{Mg}^{(56)}, \mathrm{K}$, low $\mathrm{Na}^{(57)}$ and sugar $^{(58)}$. The potential mechanisms of action between the individual components of the DASH diet pattern and cardiometabolic health have been extensively described in a recent review ${ }^{(59)}$. However, 'food synergy' is the basis of dietary pattern research. 'Food synergy' is the theory that the interaction between chemical compounds within the diet may have additive effects on physiology ${ }^{(60)}$. The potential nutrient-nutrient interactions and synergistic impact of DASH dietary components on CVD are potentially important and requires further investigation.

Four scores, Nordic, O-Med, DRV and HDI were found to have lower levels and numbers of associations with CVD risk markers compared with the American-derived scores. The Nordic and O-Med scores were developed based on dietary patterns specific to geographically defined population groups, suggesting that the American scores more adequately capture the foods consumed in the UK compared with traditional Nordic and South Mediterranean dietary intake. The O-Med was modified to A-Med for application to an American population sample; total cereals were replaced with whole grains, potato was removed from the vegetable category and dairy was excluded ${ }^{(22)}$. The only score we tested that was developed for the UK population was the DRV score. In contrast to a previous study, we did not observe associations between a higher DRV score with central adiposity, total cholesterol or markers of glucose metabolism ${ }^{(25)}$. These differences may reflect the demographic of our cohort, female compared with a younger predominately male cohort and the different outcome measures for these risk factors (waist circumference and HbA1c). It has been previously suggested that dietary scores should be developed and applied based on the target study population ${ }^{(13)}$; therefore, the lack of associations between the UK DRV score and CVD risk markers is contrary to what we expected. The DRV and HDI (developed from WHO dietary guidelines) had a low number of associations with markers of CVD risk and low-moderate correlation coefficients with other scores. Both of these scores are primarily derived from nutrient intakes rather 

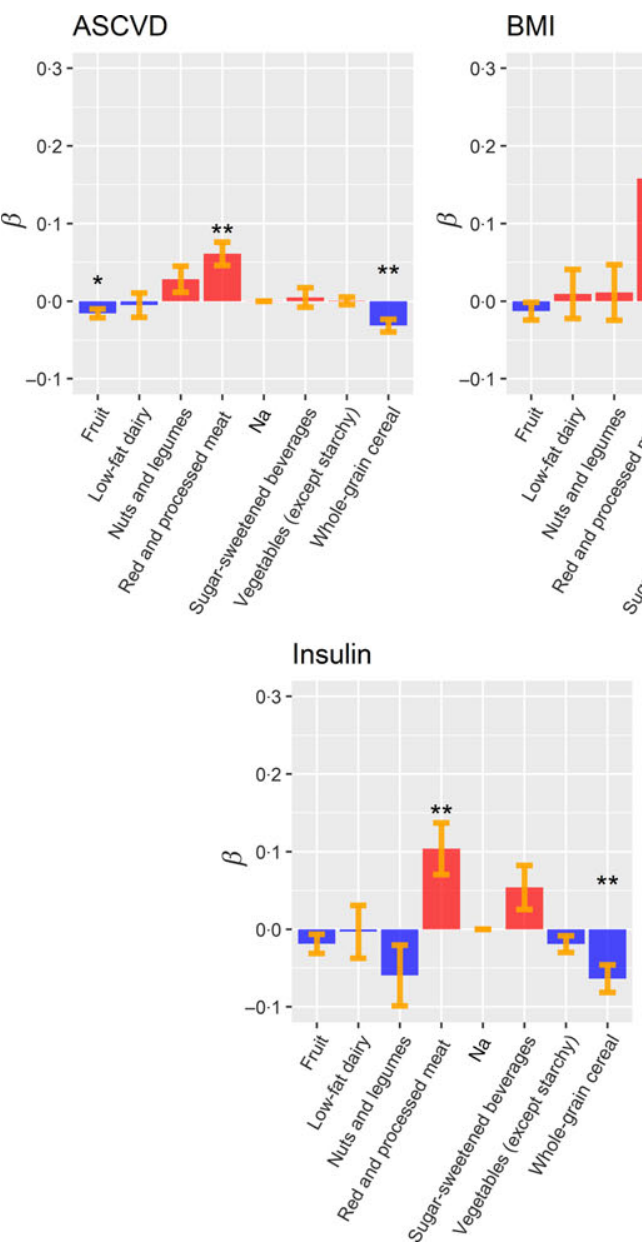

BMI

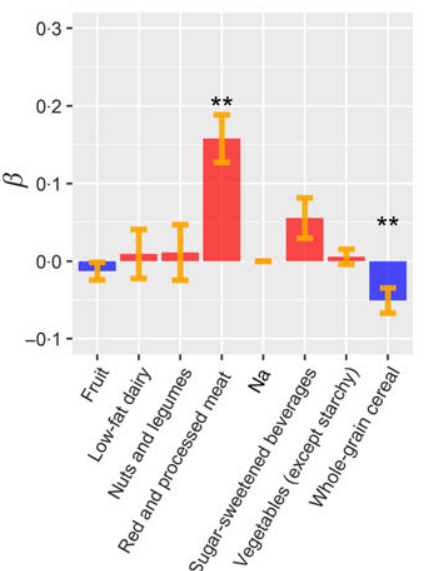

HDL

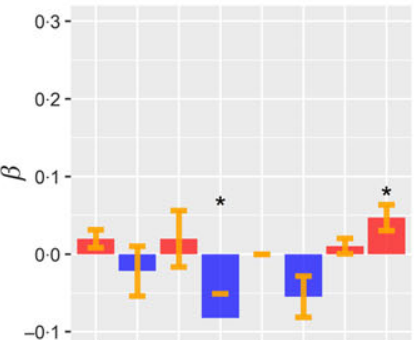

$-0 \cdot 1$

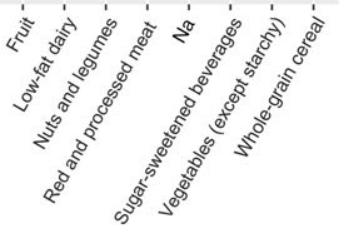

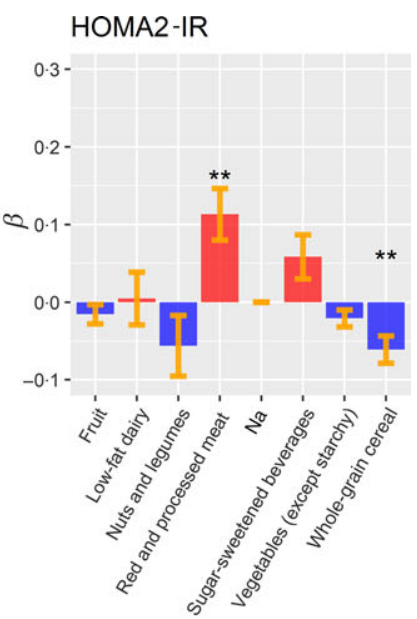

TAG

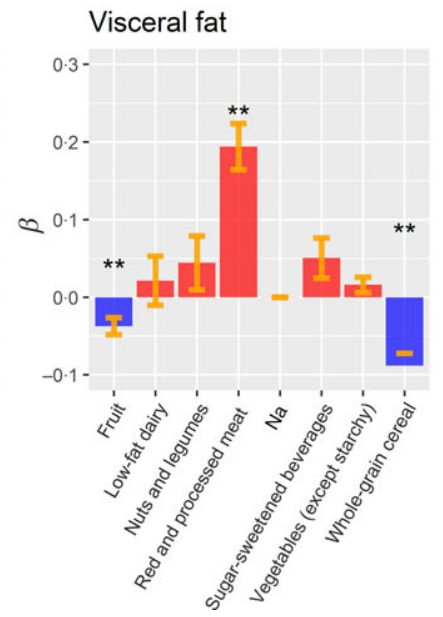

Fig. 3. Box plots representing the effects $(\beta)$ of Dietary Approaches to Stop Hypertension (DASH) food groups/nutrients on the associated CVD risk factors. The colour indicates a positive (blue) or negative (red) impact on the CVD risk factors. Standard errors are depicted in orange. To compare the effect of DASH food groups/nutrients, CVD risk factors were standardised ( $z$-score) before the analysis. Linear mixed models Benjamini and Hochberg-adjusted $P$ values are marked with asterisks $\left({ }^{\star} P_{\mathrm{FDR}}<0.05 ;{ }^{* \star} P_{\mathrm{FDR}}<0.01\right)$. ASCVD, atherosclerotic CVD; HOMA2-IR, homoeostasis model assessment of insulin resistance.

than foods ${ }^{(25,26)}$. Unlike the scores that are based on food group intake, they are not able to capture the complexity of overall diet intake $^{(61)}$; these scores also apply an absolute, rather than relative, scoring system. For example, the DRV score does not separate fruit from vegetables with $>420 \mathrm{~g}$ of fruit and vegetables per $\mathrm{d}$ (approximately five servings) obtaining the maximum score, whereas $<380$ would get a score of 0 (approximately four servings $)^{(25)}$. Similarly, the HDI awards a score of 0 for fruit and vegetables if the consumption is below $400 \mathrm{~g} / \mathrm{d}$, not allowing the differentiation between individuals.

In our post hoc investigation, to determine if any specific food groups were driving associations between the DASH score and markers of cardiometabolic risk, we found that higher intakes of wholegrains were beneficially associated with all CVD markers. Additionally, higher fruit intake was associated lower with TAG and VF mass, while red/processed meat associated with higher insulin, HOMA2-IR, VAT, BMI, TAG and ASCVD. The observed determinantal effect of red meat on glucose metabolism is in agreement with pooled analyses from the Health Professionals and Nurses' Health Study that observed about 20 and about
$50 \%$ increase in type 2 diabetes risk per $100 \mathrm{~g} / \mathrm{red}$ meat and processed meat intake per $\mathrm{d}^{(62)}$. Although a causal relationship needs to be established, suggested physiological mechanisms linking red meat and type 2 diabetes focus around inflammatory pathways induced by Fe, nitrosamines and advanced glycation end products that are associated with red and processed meat intake ${ }^{(63)}$. Higher whole-grain intake has previously been associated with lower $\operatorname{VAT}^{(64)}$. Whole grains and fruit are an important contributor to dietary fibre in the UK population, fibre from these food groups has been previously associated with lower adiposity ${ }^{(65)}$. Potential mechanisms include the non-nutrient components, for example, polyphenol intake linked to these food groups ${ }^{(66)}$ and bacterial fermentation in the colon of SCFA impacting on various metabolic pathways related to cardiometabolic health $^{(67)}$.

The main strength of the study is the breadth of objectively measured CVD-related risk markers on a large UK population sample. This combined with the dietary data obtained from a validated FFQ has allowed us to test associations from the main dietary scores with these markers. We focused on a female 
population sample as there is a need for a targeted approach in understanding the differential CVD risk in women, of which diet may be an important modifiable factor. The generalisability of our observations is therefore, limited to women. Due to the application of scoring systems that are based on population sample distribution of intakes, our results may also not be applicable to women with different demographics to the TwinsUK cohort. In UK cohorts, the DASH dietary pattern has been associated with lower odds of the metabolic syndrome in the Airwave Health Monitoring study, a predominately male cohort of police force employees ${ }^{(68)}$ and A-HEI-2010 applied to the Whitehall II cohort reported positive associations with CVD-free life expectancy ${ }^{(69)}$. However, these are occupational-specific cohorts testing single scores; therefore, further research is needed to compare associations between sexes to establish the likely importance of sex-stratified dietary recommendations for CVD prevention in the UK. Additional limitation to this study may be the gap (on average 2.6 years) between the clinical assessment and the FFQ. However, longitudinal studies showed that patterns of dietary intake are established during childhood and maintained into adulthood ${ }^{(15,70,71)}$. Therefore, it is unlikely that the small difference between the time FFQ and the collection of the clinical phenotypes would substantially affect the results reported in this study. Finally, our study includes the inherent limitations of all self-report retrospective dietary assessment methods of recall bias and misreporting of dietary intake. However, the FFQ used in the TwinsUK study to estimate habitual diet is based on the validated FFQ developed for the European Prospective Investigation into Diet and Cancer study ${ }^{(17)}$ and has been shown to detect associations between diet exposure and cardiometabolic health outcomes in UK population samples ${ }^{(69,72)}$. High $\mathrm{Na}$ intake is established risk factor for elevated blood pressure ${ }^{(73)}$. FFQ do not capture discretionary salt which is likely to contribute to the poor agreement between FFQ estimated intake with 24-h urine ('gold standard' measurement ${ }^{(74)}$. Therefore, $\mathrm{Na}$ intake is likely to be systematically underestimated in the scores that include $\mathrm{Na}$ or salt, leading to potential attenuation of observed associations. Last, the crosssectional design of our study can only inform associations for further testing.

In conclusion, the present study suggests that in the female UK population, the DASH diet is associated with a more favourable CVD risk profile. Increasing whole-grain cereal and reducing red meat intake may confer specific benefit as part of this dietary pattern. Currently, specified dietary patterns are not part of UK dietary guidelines. Although our observations require replication and testing, the DASH dietary pattern could be a candidate dietary pattern to supplement current UK dietary recommendations for CVD prevention.

\section{Acknowledgements}

We are grateful to the twins who took part in TwinsUK and the whole TwinsUK team, which includes academic researchers, clinical staff, laboratory technicians, administrative staff and research managers.
TwinsUK receives funding from the Wellcome Trust (212904/ Z/18/Z), Medical Research Council (AIMHY; MR/M016560/1) and European Union (H2020 contract no. 733100). TwinsUK and M. M. are supported by the National Institute for Health Research (NIHR)-funded BioResource, Clinical Research Facility and Biomedical Research Centre based at Guy's and St Thomas' NHS Foundation Trust in partnership with King's College London. O. M. is supported by Chronic Disease Research Foundation (CDRF). C. M. is funded by the Chronic Disease Research Foundation and by the Medical Research Council (MRC)/British Heart Foundation Ancestry and Biological Informative Markers for Stratification of Hypertension (AIMHY; MR/M016560/1)

O. M., S. E. B., M. M. and R. G. designed the research; O. M. and M. M. performed the main analysis; C. M. contributed to the analysis; O. M., M. M. and R. G. wrote the manuscript; O. M., S. E. B., C. M., M. M., R. G. and T. D. S. edited the manuscript. All authors read and approved the final manuscript.

T. D. S. is the co-founder of Zoe Global Ltd. S. E. B. is a consultant for Zoe Global Ltd. All other authors declare no competing financial interests.

\section{Supplementary material}

For supplementary materials referred to in this article, please visit https://doi.org/10.1017/S000711452000495X

\section{References}

1. British Heart Foundation (2020) Heart Statistics - Heart and Circulatory Diseases in the UK. https://www.bhf.org.uk/ what-we-do/our-research/heart-statistics

2. Mendelsohn ME \& Karas RH (1999) The protective effects of estrogen on the cardiovascular system. N Engl J Med 340, 1801-1811.

3. NHS Digital (2019) Health Survey for England 2018 [NS]. https:/digital.nhs.uk/data-and-information/publications/statistical/ health-survey-for-england/2018

4. Huxley R, Barzi F \& Woodward M (2006) Excess risk of fatal coronary heart disease associated with diabetes in men and women: meta-analysis of 37 prospective cohort studies. BMJ 332, 73-78.

5. Bansal S, Buring JE, Rifai N, et al. (2007) Fasting compared with nonfasting triglycerides and risk of cardiovascular events in women. JAMA 298, 309-316.

6. Nordestgaard BG, Benn M, Schnohr P, et al. (2007) Nonfasting triglycerides and risk of myocardial infarction, ischemic heart disease, and death in men and women. JAMA 298, 299-308.

7. Sharma S \& Wood MJ (2018) The global burden of cardiovascular disease in women. Curr Treat Options Cardiovasc Med 20, 81

8. Iqbal R, Anand S, Ounpuu S, et al. (2008) Dietary patterns and the risk of acute myocardial infarction in 52 countries: results of the INTERHEART study. Circulation 118, 1929-1937.

9. Hu FB (2002) Dietary pattern analysis: a new direction in nutritional epidemiology. Curr Opin Lipidol 13, 3-9.

10. Aljuraiban GS, Gibson R, Oude Griep LM, et al. (2020) Perspective: the Application of A Priori Diet Quality Scores to Cardiovascular Disease Risk - A Critical Evaluation of Current Scoring Systems. Adv Nutr 11, 10-24. 
11. US Department of Health and Human Services \& US Department of Agriculture (2015) 2015-2020 Dietary Guidelines for Americans, 8th ed. Washington, DC: US Department of Health and Human Services.

12. Cespedes EM \& Hu FB (2015) Dietary patterns: from nutritional epidemiologic analysis to national guidelines. Am J Clin Nutr 101, 899-900.

13. Waijers PM, Feskens EJ \& Ocke MC (2007) A critical review of predefined diet quality scores. Br J Nutr $\mathbf{9 7}, 219-231$.

14. Verdi S, Abbasian G, Bowyer RCE, et al. (2019) Twinsuk: the UK adult twin registry update. Twin Res Hum Genet 22, 523-529.

15. Movassagh EZ, Baxter-Jones ADG, Kontulainen S et al. (2017) Tracking dietary patterns over 20 years from childhood through adolescence into young adulthood: the Saskatchewan Pediatric Bone Mineral Accrual Study. Nutrients 9, 990.

16. Bingham SA, Gill C, Welch A, et al. (1997) Validation of dietary assessment methods in the UK arm of EPIC using weighed records, and 24-hour urinary nitrogen and potassium and serum vitamin $\mathrm{C}$ and carotenoids as biomarkers. Int $J$ Epidemiol 26, Suppl. 1, S137-S151.

17. Bingham SA, Welch AA, McTaggart A, et al. (2001) Nutritional methods in the European Prospective Investigation of Cancer in Norfolk. Public Health Nutr 4, 847-858.

18. Mulligan AA, Luben RN, Bhaniani A, et al. (2014) A new tool for converting food frequency questionnaire data into nutrient and food group values: FETA research methods and availability. BMJ Open 4, e004503.

19. Pallister T, Haller T, Thorand B, et al. (2017) Metabolites of milk intake: a metabolomic approach in UK twins with findings replicated in two European cohorts. Eur J Nutr 56, 2379-2391.

20. Frankenfield DC, Muth ER \& Rowe WA (1998) The HarrisBenedict studies of human basal metabolism. J Am Diet Assoc 98, 439-445.

21. Chiuve SE, Fung TT, Rimm EB, et al. (2012) Alternative dietary indices both strongly predict risk of chronic disease. J Nutr 142, 1009-1018.

22. Fung TT, McCullough ML, Newby PK, et al. (2005) Diet-quality scores and plasma concentrations of markers of inflammation and endothelial dysfunction. Am J Clin Nutr 82, 163-173

23. Fung TT, Chiuve SE, McCullough ML, et al. (2008) Adherence to a DASH-style diet and risk of coronary heart disease and stroke in women. Arch Intern Med 168, 713-720.

24. Huijbregts P, Feskens E, Räsänen L, et al. (1997) Dietary pattern and 20 year mortality in elderly men in Finland, Italy, and the Netherlands: longitudinal cohort study. BMJ 315, 13-17.

25. Eriksen R, Gibson R, Lamb K, et al. (2018) Nutrient profiling and adherence to components of the UK national dietary guidelines association with metabolic risk factors for CVD and diabetes: airwave Health Monitoring Study. Br J Nutr 119, 695-705.

26. Berentzen NE, Beulens JW, Hoevenaar-Blom MP, et al. (2013) Adherence to the WHO's healthy diet indicator and overall cancer risk in the EPIC-NL cohort. PLOS ONE 8, e70535.

27. Guenther PM, Casavale KO, Reedy J, et al. (2013) Update of the Healthy Eating Index: HEI-2010. J Acad Nutr Diet 113, 569-580.

28. Galbete C, Kröger J, Jannasch F, et al. (2018) Nordic diet, Mediterranean diet, and the risk of chronic diseases: the EPIC-Potsdam study. BMC Med 16, 99.

29. Trichopoulou A, Costacou T, Bamia C, et al. (2003) Adherence to a Mediterranean diet and survival in a Greek population. $N$ Engl J Med 348, 2599-2608.

30. Rizzo ML (2019) Statistical computing with R: books.google. com.

31. Jennings A, MacGregor A, Welch A, et al. (2015) Amino acid intakes are inversely associated with arterial stiffness and central blood pressure in women. J Nutr 145, 2130-2138.
32. Menni C, Gudelj I, Macdonald-Dunlop E, et al. (2018) Glycosylation profile of immunoglobulin $\mathrm{g}$ is cross-sectionally associated with cardiovascular disease risk score and subclinical atherosclerosis in two independent cohorts. Circ Res 122, 1555-1564.

33. Menni C, Migaud M, Glastonbury CA, et al. (2016) Metabolomic profiling to dissect the role of visceral fat in cardiometabolic health. Obesity (Silver Spring) 24, 1380-1388.

34. Ehret GB, Munroe PB, Rice KM, et al. (2011) Genetic variants in novel pathways influence blood pressure and cardiovascular disease risk. Nature 478, 103-109.

35. Jamshidi Y, Snieder H, Wang X, et al. (2006) Common polymorphisms in SOCS3 are not associated with body weight, insulin sensitivity or lipid profile in normal female twins. Diabetologia 49, 306-310.

36. American College of Cardiology (2020) ASCVD risk estimator. https://tools.acc.org/ldl/ascvd_risk_estimator/index. html\#!/calulate/estimator/

37. Pain O, Dudbridge F \& Ronald A (2018) Are your covariates under control? How normalization can re-introduce covariate effects. Eur J Hum Genet 26, 1194-1201.

38. Bates D, Mächler M, Bolker B, et al. (2015) Fitting linear mixed-effects models using lme4. J Stat Softw 67, 1-48.

39. Kim S, Haines PS, Siega-Riz AM, et al. (2003) The Diet Quality Index-International (DQI-I) provides an effective tool for cross-national comparison of diet quality as illustrated by China and the United States. J Nutr 133, 3476-3484.

40. Miller PE, Cross AJ, Subar AF, et al. (2013) Comparison of 4 established DASH diet indexes: examining associations of index scores and colorectal cancer. Am J Clin Nutr $\mathbf{9 8}$, 794-803.

41. Saneei P, Salehi-Abargouei A, Esmaillzadeh A, et al. (2014) Influence of Dietary Approaches to Stop Hypertension (DASH) diet on blood pressure: a systematic review and meta-analysis on randomized controlled trials. Nutr Metab Cardiovasc Dis 24, 1253-1261.

42. Benjamini Y \& Hochberg Y (1995) Controlling the false discovery rate: A practical and powerful approach to multiple testing. J Roy Stat Soc B (Methodol) 57, 289-300

43. Lee LV \& Foody JM (2008) Cardiovascular disease in women. Curr Atheroscler Rep 10, 295-302.

44. Oterdoom LH, de Vries APJ, Gansevoort RT, et al. (2009) Fasting insulin is a stronger cardiovascular risk factor in women than in men. Atherosclerosis 203, 640-646.

45. Tohidi M, Baghbani-Oskouei A, Ahanchi NS, et al. (2018) Fasting plasma glucose is a stronger predictor of diabetes than triglyceride-glucose index, triglycerides/high-density lipoprotein cholesterol, and homeostasis model assessment of insulin resistance: tehran Lipid and Glucose Study. Acta Diabetol 55, 1067-1074.

46. Liese AD, Krebs-Smith SM, Subar AF, et al. (2015) The Dietary Patterns Methods Project: synthesis of findings across cohorts and relevance to dietary guidance. J Nutr 145, 393-402.

47. Aune D, Giovannucci E, Boffetta P, et al. (2017) Fruit and vegetable intake and the risk of cardiovascular disease, total cancer and all-cause mortality-a systematic review and doseresponse meta-analysis of prospective studies. Int J Epidemiol 46, 1029-1056.

48. Mellen PB, Walsh TF \& Herrington DM (2008) Whole grain intake and cardiovascular disease: a meta-analysis. Nutr Metab Cardiovasc Dis 18, 283-290.

49. Salehi-Abargouei A, Maghsoudi Z, Shirani F, et al. (2013) Effects of Dietary Approaches to Stop Hypertension (DASH)-style diet on fatal or nonfatal cardiovascular diseases - incidence: a systematic review and meta-analysis on observational prospective studies. Nutrition 29, 611-618. 
50. Chiu S, Bergeron N, Williams PT, et al. (2016) Comparison of the DASH (Dietary Approaches to Stop Hypertension) diet and a higher-fat DASH diet on blood pressure and lipids and lipoproteins: a randomized controlled trial. Am J Clin Nutr 103, 341-347.

51. Kucharska A, Gajewska D, Kiedrowski M, et al. (2018) The impact of individualised nutritional therapy according to DASH diet on blood pressure, body mass, and selected biochemical parameters in overweight/obese patients with primary arterial hypertension: a prospective randomised study. Kardiol Pol 76, 158-165.

52. Ehrampoush E, Nazari N, Homayounfar R, et al. (2020) Association between dietary patterns with insulin resistance in an Iranian population. Clin Nutr ESPEN 36, 45-52.

53. Siervo M, Lara J, Chowdhury S, et al. (2015) Effects of the Dietary Approach to Stop Hypertension (DASH) diet on cardiovascular risk factors: a systematic review and meta-analysis. Br J Nutr 113, 1-15.

54. Barrett EM, Batterham MJ, Ray S, et al. (2019) Whole grain, bran and cereal fibre consumption and CVD: a systematic review. $\mathrm{Br}$ J Nutr 121, 914-937.

55. Asemi Z, Saneei P, Sabihi SS, et al. (2015) Total, dietary, and supplemental calcium intake and mortality from all-causes, cardiovascular disease, and cancer: A meta-analysis of observational studies. Nutr Metab Cardiovasc Dis 25, 623-634.

56. Houston M (2011) The role of magnesium in hypertension and cardiovascular disease. J Clin Hypertens (Greenwich) 13, 843-847.

57. Newberry SJ, Chung M, Anderson CAM, et al. (2018) Sodium and Dietary Patterns Over 20 Years from Childhood Through Adolescence into Young Adulthood: Potassium Intake: Effects on Chronic Disease Outcomes and Risks, AHRQ Comparative Effectiveness Reviews. Rockville, MD: Agency for Healthcare Research and Quality (US).

58. Bergwall S, Ramne S, Sonestedt E, et al. (2019) High versus low added sugar consumption for the primary prevention of cardiovascular disease. Cochrane Database Syst Rev, issue 4, CD013320.

59. Akhlaghi M (2019) Dietary Approaches to Stop Hypertension (DASH): potential mechanisms of action against risk factors of the metabolic syndrome. Nutr Res Rev 33, 1-18.

60. Jacobs DR, Jr, Gross MD \& Tapsell LC (2009) Food synergy: an operational concept for understanding nutrition. Am J Clin Nutr 89, 1543S-1548S.

61. Arvaniti F \& Panagiotakos DB (2008) Healthy indexes in public health practice and research: a review. Crit Rev Food Sci Nutr 48, 317-327.

62. Pan A, Sun Q, Bernstein AM, et al. (2011) Red meat consumption and risk of type 2 diabetes: 3 cohorts of US adults and an updated meta-analysis. Am J Clin Nutr 94, 1088-1096.
63. Tong M, Neusner A, Longato L, et al. (2009) Nitrosamine exposure causes insulin resistance diseases: relevance to type 2 diabetes mellitus, non-alcoholic steatohepatitis, and Alzheimer's disease. J Alzheimers Dis 17, 827-844.

64. McKeown NM, Troy LM, Jacques PF, et al. (2010) Whole- and refined-grain intakes are differentially associated with abdominal visceral and subcutaneous adiposity in healthy adults: the Framingham Heart Study. Am J Clin Nutr 92, $1165-1171$.

65. Gibson R, Eriksen R, Chambers E, et al. (2019) Intakes and food sources of dietary fibre and their associations with measures of body composition and inflammation in UK adults: crosssectional analysis of the Airwave Health Monitoring Study. Nutrients 11, 1839.

66. Guo X, Tresserra-Rimbau A, Estruch R, et al. (2017) Polyphenol levels are inversely correlated with body weight and obesity in an elderly population after 5 years of follow up (the randomised PREDIMED study). Nutrients $9,452$.

67. Vetrani C, Costabile G, Luongo D, et al. (2016) Effects of whole-grain cereal foods on plasma short chain fatty acid concentrations in individuals with the metabolic syndrome. Nutrition 32, 217-221.

68. Gibson R, Eriksen R, Singh D, et al. (2018) A cross-sectional investigation into the occupational and socio-demographic characteristics of British police force employees reporting a dietary pattern associated with cardiometabolic risk: findings from the Airwave Health Monitoring Study. Eur J Nutr 57, 2913-2926.

69. Lagström H, Stenholm S, Akbaraly T, et al. (2020) Diet quality as a predictor of cardiometabolic disease-free life expectancy: the Whitehall II cohort study. Am J Clin Nutr 111, 787-794.

70. Lien N, Lytle LA, Klepp K-I (2001) Stability in consumption of fruit, vegetables, and sugary foods in a cohort from age 14 to Age 21. Prev Med 33, 217-226.

71. Mikkilä V, Räsänen L, Raitakari OT, et al. (2007) Consistent dietary patterns identified from childhood to adulthood: the Cardiovascular Risk in Young Finns Study. Br J Nutr 93, 923-931.

72. Brunner E, Stallone D, Juneja M, et al. (2001) Dietary assessment in Whitehall II: comparison of $7 \mathrm{~d}$ diet diary and food-frequency questionnaire and validity against biomarkers. Br J Nutr 86, 405-414.

73. Mozaffarian D, Fahimi S, Singh GM, et al. (2014) Global sodium consumption and death from cardiovascular causes. $N$ Engl J Med 371, 624-634.

74. McLean RM, Farmer VL, Nettleton A, et al. (2017) Assessment of dietary sodium intake using a food frequency questionnaire and 24-hour urinary sodium excretion: a systematic literature review. J Clin Hypertens (Greenwich) 19, 1214-1230. 